\title{
Modified Stable Election Protocol (M-SEP) for Hierarchical WSN
}

\author{
Greeshma Arya \\ Department of Electronics Engineering \\ Indira Gandhi Delhi Technical University \\ Delhi, India
}

\author{
D S Chauhan \\ Department of Electrical Engineering \\ Uttarakhand Technical University \\ Dehradun,India
}

\begin{abstract}
In this paper, M-SEP (Modified Stable Election Protocol) is proposed for electing cluster heads in a distributed fashion in two, three and up to ninth level hierarchical wireless sensor networks. The significant improvement has been seen using MSEP in comparison with SEP in terms of network lifetime, energy consumption and data transmission to BS. The simulation results are compared and analysis is done to see the scope of future work. The superior characteristic of this protocol is discussed by describing the probability equations for multi level M-SEP. Here two aggregators are introduced along with one sink in the centre. The results reveal that there is $200 \%$ improvement in the overall lifetime of the network by using M-SEP after comparing two-level, three-level and up to ninth level network hierarchy. Two aggregators are placed at different coordinates and results are compiled. This work also recommends a final placement of the gathering nodes for best results. The investigations ascertain that network lifetime and efficiency increases by using M-SEP over SEP.
\end{abstract}

\section{General Terms}

Wireless sensor network, Network lifetime, Cluster, Heterogeneous network.

\section{Keywords}

Aggregators, Gathering Nodes, Super nodes, advanced nodes, super advanced nodes.

\section{INTRODUCTION}

SEP: A Stable Election Protocol for clustered heterogeneous wireless sensor networks

SEP [2] is a scheme/protocol for heterogeneous wireless sensor networks. It assumes that a percentage of the population of sensor nodes is equipped with additional energy resources this is a source of heterogeneity which may result from the initial setting or as the operation of the network evolves. Classical clustering protocols assume that all the nodes are equipped with the same amount of energy and as a result, they cannot take full advantage of the presence of node heterogeneity. Here two types of nodes (Advanced and normal nodes) are considered with different initial energy. The advanced nodes are equipped with more energy than the normal nodes at the beginning. . SEP is basically based on weighted election probabilities of each node to become cluster head according to the remaining energy in each node. It has been observed that the SEP yields longer stability region for higher values of extra energy brought by more powerful nodes, but it cannot be applied to multi-level heterogeneous WSNs.

It assumes the case where a percentage of the population of sensor nodes is equipped with more energy resources than the rest of the nodes. Let $m$ be the fraction of the total number of nodes $\mathrm{n}$, which are equipped with $\alpha$ times more energy than the others. We refer to these powerful nodes as advanced nodes, and the rest $(1-m) \times n$ as normal nodes. It is assumed that all nodes are distributed uniformly over the sensor field.

In order to prolong the stable region, SEP attempts to maintain the constraint of well balanced energy consumption. Intuitively, advanced nodes have to become cluster heads more often than the normal nodes, which is equivalent to a fairness constraint on energy consumption. The total energy of the system changes unequally. Suppose that $E_{0}$ is the initial energy of each normal sensor. The energy of each advanced node is then $\mathrm{E}_{0}(1+\alpha)$.The total (initial) energy of the new heterogeneous setting is equal to [2]:

$$
n \cdot(1-m) \cdot E_{o}+n \cdot m \cdot E_{o} \cdot(1+\propto)=n \cdot E_{o} \cdot(1+\propto \cdot m)
$$

Where

Eo- initial energy of a normal sensor node $\mathrm{m}$ - Fraction of the total number of nodes $\alpha$ - amount of extra energy with advanced nodes

So, the total energy of the system is increased by a factor of $(1+\alpha \times \mathrm{m}$.

It was observed that the stable region of SEP is extended compared to that of LEACH, even though the gain is not very large. Moreover, the unstable region of SEP is shorter than that of LEACH. This is because under SEP, the advanced nodes follow the death process of normal nodes, as the weighted probability of electing cluster heads causes the energy of each node to be consumed in proportion to the node's initial energy.

\section{PERFORMANCE MEASURING FACTORS}

These are parameters used in this performance evaluation of clustering protocol [1]

Stability Period: is the time interval from the start of operation until the death of the first sensor node. We also refer to this period as "stable region."

Instability Period: is the time interval from the death of the first node until the death of the last sensor node. We also refer to this period as "unstable region [4]."

Network lifetime: is the time interval from the start of operation (of the sensor network) until the death of the last alive node.

Number of cluster heads per round: This instantaneous measure reflects the number of nodes which would send directly to the sink information aggregated from their cluster members. 
Number of alive (total, advanced and normal) nodes per round: This instantaneous measure reflects the total number of nodes and that of each type that has not yet expended all of their energy.

Throughput: We measure the total rate of data sent over the network, the rate of data sent from cluster heads to the sink as well as the rate of data sent from the nodes to their cluster heads.

Clearly, the larger the stable region and the smaller the unstable region are, the better the reliability of the clustering process of the sensor network is. On the other hand, there is a tradeoff between reliability and the lifetime of the system. Until the death of the last node we can still have some feedback about the sensor field even though this feedback may not be reliable. The unreliability of the feedback stems from the fact that there is no guarantee that there is at least one cluster head per round during the last rounds of the operation.

In this model, the absence of a cluster head in an area prevents any reporting about that area to the sink. The throughput measure captures the rate of such data reporting to the sink.

\section{MODEL OF PROPOSED PROTOCOL (M-SEP)}

In this section the model of a wireless sensor network with nodes heterogeneous in their initial amount of energy is described. Heterogeneous means that the nodes have different processing capabilities. The heterogeneous model yields higher coverage range and lower cost for the same deployable sensor nodes.

Here the network is chosen as heterogeneous in nature. So some nodes are equipped with more energy than the rest of the nodes. Two aggregators are introduced on different sides of the sink and changed their positions for nine levels of heterogeneity and compared the lifetime and efficiency of the model in each case. It is observed that as the number of levels increased, there was an improvement in most of the parameters, such as the first dead node, the last dead node, the lifetime of the network etc. For Example Level 1 had advanced nodes and normal nodes (same as SEP). The advanced nodes had $\alpha$ time more energy than the normal nodes. Level 2 had normal, advanced and super advanced nodes and so on.

Apart from this, using two aggregators significantly reduced the energy consumption of the nodes. The nodes will send their data to the cluster head and the cluster heads will send the data to the sink or any of the aggregators, whichever is nearer. The cluster heads will calculate the distance from sink and the aggregator. The aggregators after collecting the data from the cluster heads transfer the data to the sink and are considered to have infinite energy. No processing of data takes place at the aggregator unlike sink.

M-SEP protocol goal is to increase the lifetime and stability of the network in the presence of heterogeneous nodes. Since cluster heads consume more energy than cluster members in receiving and sensing data from their member nodes, performing signal processing and sending the aggregated data to next node or base station, the role of cluster head must be rotated among sensor nodes. Therefore, M-SEP works in rounds as SEP and also considers how to optimally select the cluster heads in the heterogonous network. Traditionally as per SEP, Cluster head algorithm is broken into rounds. At each round node decides whether to become a cluster head based on threshold calculated by the suggested percentage of cluster heads for the network (determined a priori) and the number of times the node has been a cluster-head so far. This decision is made by the nodes by choosing the random number between 0 and 1 . If the number is less than a threshold $\mathrm{T}(\mathrm{si})$ the node becomes a cluster-head for the current round. In the proposed M-SEP the threshold is modified and set as:

temp_rand $=(\mathrm{p} /(1-\mathrm{p} * \bmod (\mathrm{r}, \operatorname{round}(1 / \mathrm{p})))$

Where ' $p$ ' is the probability of a node to be selected as a cluster head in the current round. Hence, threshold is set differently and dependent on that has been set according to two-, threeand multilevel heterogeneity as mentioned below.

For the purpose of this study, we use similar radio communication and consumption model as reported in SEP.

\section{Aggregators:}

Two Aggregators are placed on the left and right side of Sink. Using two aggregators significantly reduced the energy consumption of the nodes. The nodes will send their data to the cluster head and the cluster heads will send the data to the sink or any of the aggregators, whichever is nearer. The cluster heads will calculate the distance from sink and the aggregators. The aggregators after collecting the data from the cluster heads transfer the data to the sink and are considered to have infinite energy. No processing of data takes place at the aggregators unlike sink.

The data for different positions of the aggregators is compared for different levels of heterogeneity. The three cases for placement of aggregators are as follows:
1) $(25,50)$ Sink $(75,50)$
2) $(15,50)$ Sink $(85,50)$
3) $(35,50)$ Sink $(65,50)$

\section{Two-level Heterogeneity}

Two type of nodes known as normal and advanced nodes are considered with their different initial energy for two-level heterogeneous networks. The reference value of $p$ is different for these types of nodes. The probabilities of normal and advanced nodes are obtained similarly as reported in SEP:

Election Probability for Normal Nodes

$\mathrm{p}_{\mathrm{nrm}}=(\mathrm{p} /(1+\mathrm{a} * \mathrm{~m}))$

Election Probability for Advanced Nodes

$\mathrm{p}_{\mathrm{adv}}=(\mathrm{p} *(1+\mathrm{a}) /(1+\mathrm{a} * \mathrm{~m}))$

\section{Three-level Heterogeneity}

In this case three types of nodes known as normal, advanced and super nodes are considered based on fractional difference in their initial energy level. Here the reference value of $p$ is different for these types of nodes. The probabilities of normal, advanced and super nodes are:

Election Probability for Normal Nodes

$\mathrm{p}_{\mathrm{nrm}}=(\mathrm{p} /(1+(\mathrm{m} *(\mathrm{a}+(\mathrm{mo} * \mathrm{~b})))))$

Election Probability for Advanced Nodes

$\mathrm{p}_{\mathrm{adv}}=((\mathrm{p} *(1+\mathrm{a})) /(1+(\mathrm{m} *(\mathrm{a}+(\mathrm{mo} * \mathrm{~b})))))$

$\mathrm{P}_{\mathrm{sup}}==((\mathrm{p} *(1+\mathrm{b})) /(1+(\mathrm{m} *(\mathrm{a}+(\mathrm{mo} * \mathrm{~b})))))$

Four-level Heterogeneity

Four types of nodes known as normal, advanced super supersuper nodes are considered with their different initial energy 
for four-level heterogeneous networks. The reference value of $p$ is different for these types of nodes. The probabilities of these four nodes are:

Election Probability for Normal Nodes

$\mathrm{p}_{\mathrm{nrm}}=(\mathrm{p} /(1+(\mathrm{m} *(\mathrm{a}+(\mathrm{mo} * \mathrm{~b})+(\mathrm{mp} * \mathrm{c})))))$

Election Probability for Advanced Nodes

$\mathrm{p}_{\mathrm{adv}}=((\mathrm{p} *(1+\mathrm{a})) /(1+(\mathrm{m} *(\mathrm{a}+(\mathrm{mo} * \mathrm{~b})+(\mathrm{mp} * \mathrm{c})))))$

$\mathrm{p}_{\text {sup }}=((\mathrm{p} *(1+\mathrm{b})) /(1+(\mathrm{m} *(\mathrm{a}+(\mathrm{mo} * \mathrm{~b})+(\mathrm{mp} * \mathrm{c})))))$

$\mathrm{p}_{\text {sup } 4}=((\mathrm{p} *(1+\mathrm{c})) /(1+(\mathrm{m} *(\mathrm{a}+(\mathrm{mo} * \mathrm{~b})+(\mathrm{mp} * \mathrm{c})))))$

Similarly the heterogeneity is increased upto $9^{\text {th }}$ level and the probabilities are as follows:

\section{Fifth level Heterogeneity:}

$\mathrm{p}_{\text {nrm }}=(\mathrm{p} /(1+(\mathrm{m} *(\mathrm{a}+(\mathrm{mo} * \mathrm{~b})+(\mathrm{mp} * \mathrm{c})+(\mathrm{mq} * \mathrm{~d})))))$

Election Probability for Advanced Nodes

$\mathrm{p}_{\mathrm{adv}}=((\mathrm{p} *(1+\mathrm{a})) /(1+(\mathrm{m} *(\mathrm{a}+(\mathrm{mo} * \mathrm{~b})+(\mathrm{mp} * \mathrm{c})+(\mathrm{mq} * \mathrm{~d})))))$

$\mathrm{p}_{\text {sup }}=\left((\mathrm{p} *(1+\mathrm{b})) /\left(1+\left(\mathrm{m}^{*}(\mathrm{a}+(\mathrm{mo} * \mathrm{~b})+(\mathrm{mp} * \mathrm{c})+(\mathrm{mq} * \mathrm{~d}))\right)\right)\right)$

$\mathrm{p}_{\text {sup } 5}=\left((\mathrm{p} *(1+\mathrm{d})) /\left(1+\left(\mathrm{m} *\left(\mathrm{a}+(\mathrm{mo} * \mathrm{~b})+(\mathrm{mp} * \mathrm{c})+\left(\mathrm{mq}^{*} \mathrm{~d}\right)\right)\right)\right)\right)$;

\section{Sixth level Heterogeneity:}

$\mathrm{p}_{\text {nrm }}=(\mathrm{p} /(1+(\mathrm{m} *(\mathrm{a}+(\mathrm{mo} * \mathrm{~b})+(\mathrm{mp} * \mathrm{c})+(\mathrm{mq} * \mathrm{~d})+(\mathrm{mr} * \mathrm{e})))))$

Election Probability for Advanced Nodes

$\mathrm{p}_{\mathrm{adv}}=(\quad(\mathrm{p} *(1+\mathrm{a})) /(1+(\mathrm{m} *(\mathrm{a}+(\mathrm{mo} * \mathrm{~b})+(\mathrm{mp} * \mathrm{c})+\quad(\mathrm{mq} * \mathrm{~d})+$ $(\mathrm{mr} * \mathrm{e}))))$

$\mathrm{p}_{\text {sup }}=\left(\left(\mathrm{p}^{*}(1+\mathrm{b})\right) /(1+(\mathrm{m} *(\mathrm{a}+(\mathrm{mo} * \mathrm{~b})+(\mathrm{mp} * \mathrm{c})+(\mathrm{mq} * \mathrm{~d})+\right.$ $(\mathrm{mr} * \mathrm{e})))))$

$\mathrm{p}_{\text {sup } 5}=((\mathrm{p} *(1+\mathrm{d})) /(1+(\mathrm{m} *(\mathrm{a}+(\mathrm{mo} * \mathrm{~b})+(\mathrm{mp} * \mathrm{c})+(\mathrm{mq} * \mathrm{~d})+$

$(\mathrm{mr} * \mathrm{e})))))$;

$\mathrm{p}_{\text {sup }}=((\mathrm{p} *(1+\mathrm{e})) /(1+(\mathrm{m} *(\mathrm{a}+(\mathrm{mo} * \mathrm{~b})+(\mathrm{mp} * \mathrm{c})+(\mathrm{mq} * \mathrm{~d})+(\mathrm{mr} * \mathrm{e}))))$ );

Similarly it is done for $7^{\text {th }}$ and $8^{\text {th }}$ level. For $9^{\text {th }}$ level as follows;

\section{Ninth level Heterogeneity:}

$\mathrm{p}_{\mathrm{nrm}}=(\mathrm{p} /(1+(\mathrm{m} *(\mathrm{a}+(\mathrm{mo} * \mathrm{~b})+(\mathrm{mp} * \mathrm{c})+(\mathrm{mq} * \mathrm{~d})+(\mathrm{mr} * \mathrm{e})+(\mathrm{ms} * \mathrm{f})+($ $\left.\left.\left.\left.\left.m t^{* g}\right)+(m u * h)\right)\right)\right)\right)$

Election Probability for Advanced Nodes

$\mathrm{p}_{\mathrm{adv}}=(\quad(\mathrm{p} *(1+\mathrm{a})) /(1+(\mathrm{m} *(\mathrm{a}+(\mathrm{mo} * \mathrm{~b})+(\mathrm{mp} * \mathrm{c})+\quad(\mathrm{mq} * \mathrm{~d})+$ $\left.\left.\left.\left(m r^{*} \mathrm{e}\right)+(\mathrm{ms} * \mathrm{f})+(\mathrm{mt} * \mathrm{~g})+(\mathrm{mu} * \mathrm{~h})\right)\right)\right)$

$\mathrm{p}_{\text {sup }}=((\mathrm{p} *(1+\mathrm{b})) /(1+(\mathrm{m} *(\mathrm{a}+(\mathrm{mo} * \mathrm{~b})+(\mathrm{mp} * \mathrm{c})+(\mathrm{mq} * \mathrm{~d})+$ $(\mathrm{mr} * \mathrm{e})+(\mathrm{ms} * \mathrm{f})+(\mathrm{mt} * \mathrm{~g})+(\mathrm{mu} * \mathrm{~h})))))$

$\mathrm{p}_{\text {sup5 }}=((\mathrm{p} *(1+\mathrm{d})) /(1+(\mathrm{m} *(\mathrm{a}+(\mathrm{mo} * \mathrm{~b})+(\mathrm{mp} * \mathrm{c})+(\mathrm{mq} * \mathrm{~d})+(\mathrm{mr} * \mathrm{e})+$ $(\mathrm{ms} * \mathrm{f})+(\mathrm{mt} * \mathrm{~g})+(\mathrm{mu} * \mathrm{~h})))))$; $\mathrm{p}_{\text {sup } 6}=\left(\left(\mathrm{p}^{*}(1+\mathrm{e})\right) /\left(1+\left(\mathrm{m} *\left(\mathrm{a}+(\mathrm{mo} * \mathrm{~b})+\left(\mathrm{mp}^{*} \mathrm{c}\right)+(\mathrm{mq} * \mathrm{~d})+(\mathrm{mr} * \mathrm{e})+\right.\right.\right.\right.$ $\left.\left.\left.\left.\left(m *^{*}\right)+\left(m t^{* g}\right)+(m u * h)\right)\right)\right)\right)$;

$\mathrm{p}_{\text {sup }}=((\mathrm{p} *(1+\mathrm{f})) /(1+(\mathrm{m} *(\mathrm{a}+(\mathrm{mo} * \mathrm{~b})+(\mathrm{mp} * \mathrm{c})+(\mathrm{mq} * \mathrm{~d})+(\mathrm{mr} * \mathrm{e})+($ $\mathrm{ms} * \mathrm{f})+(\mathrm{mt} * \mathrm{~g})+(\mathrm{mu} * \mathrm{~h})))))$;

$\mathrm{p}_{\text {sup } 8}=((\mathrm{p} *(1+\mathrm{g})) /(1+(\mathrm{m} *(\mathrm{a}+(\mathrm{mo} * \mathrm{~b})+(\mathrm{mp} * \mathrm{c})+(\mathrm{mq} * \mathrm{~d})+(\mathrm{mr} * \mathrm{e})+($ $\left.\left.\left.\left.\left.m s^{*} \mathrm{f}\right)+(\mathrm{mt} * \mathrm{~g})+(\mathrm{mu} * \mathrm{~h})\right)\right)\right)\right)$;

$\mathrm{p}_{\text {sup }}=((\mathrm{p} *(1+\mathrm{h})) /(1+(\mathrm{m} *(\mathrm{a}+(\mathrm{mo} * \mathrm{~b})+(\mathrm{mp} * \mathrm{c})+(\mathrm{mq} * \mathrm{~d})+(\mathrm{mr} * \mathrm{e})+($ $\left.\left.\left.\left.m s^{* f}\right)+(m t * g)+(m u * h)\right)\right)\right)$;

\section{SIMULATION PARAMETERS AND COMPARISON}

After repeated simulations for different levels of heterogeneity of M-SEP, the following tables are used to compare the parameters for different placement of aggregators and graphs are obtained

After Rounds $=2500$

Table 1: Parameters comparison for different heterogeneity levels for aggregators at $(25,50)$ and $(75,50)$

\begin{tabular}{|c|c|c|c|c|c|}
\hline & $\begin{array}{c}\text { Cluste } \\
\text { rs }\end{array}$ & $\begin{array}{c}\text { Dead } \\
\text { Nodes }\end{array}$ & $\begin{array}{c}\text { Alive } \\
\text { Nodes }\end{array}$ & $\begin{array}{c}\text { First } \\
\text { Dead } \\
\text { at } \\
\text { Roun } \\
\text { d }\end{array}$ & $\begin{array}{c}\text { Packe } \\
\text { ts to } \\
\text { Base } \\
\text { Statio } \\
\text { n }\end{array}$ \\
\hline SEP & 1 & 100 & 0 & 1041 & 0 \\
\hline $\begin{array}{c}\text { SEP } \\
\text { Heterogeneity } \\
\text { with 2 aggregators } \\
\text { at (25,50) and } \\
(75,50)\end{array}$ & & & & & \\
\hline 3 & 2 & 97 & 3 & 870 & 1 \\
\hline 4 & 3 & 90 & 10 & 1047 & 2 \\
\hline 5 & 15 & 71 & 29 & 1311 & 14 \\
\hline 6 & 14 & 58 & 42 & 1476 & 13 \\
\hline 7 & 28 & 43 & 57 & 1869 & 27 \\
\hline 8 & 37 & 32 & 68 & 1909 & 36 \\
\hline 9 & 21 & 21 & 79 & 1967 & 37 \\
\hline & & & & & \\
\hline
\end{tabular}

Table 2: Clusters Comparison for different placement of aggregators:

\begin{tabular}{|c|c|c|c|}
\hline SEP Heterogeneity & $\begin{array}{c}\text { Clusters } \\
\text { with 2 } \\
\text { aggregators } \\
\text { at (25,50) } \\
\text { and (75,50) }\end{array}$ & $\begin{array}{c}\text { With 2 } \\
\text { aggregators } \\
\text { at (15,50) } \\
\text { and (85,50) }\end{array}$ & $\begin{array}{c}\text { With 2 } \\
\text { aggregators } \\
\text { at (35,50) } \\
\text { and (65,50) }\end{array}$ \\
\hline 3 & 2 & 2 & 1 \\
\hline 4 & 3 & 6 & 2 \\
\hline 5 & 15 & 9 & 11 \\
\hline 6 & 14 & 18 & 17 \\
\hline 7 & 28 & 23 & 8 \\
\hline 8 & 37 & 29 & 38 \\
\hline 9 & 21 & 33 & 22 \\
\hline
\end{tabular}


Table 3: Dead nodes comparison for different placement of aggregators

\begin{tabular}{|c|c|c|c|}
\hline $\begin{array}{c}\text { SEP } \\
\text { Heterogeneity }\end{array}$ & $\begin{array}{c}\text { Dead nodes } \\
\text { with 2 } \\
\text { aggregators } \\
\text { at }(25,50) \\
\text { and }(75,50)\end{array}$ & $\begin{array}{c}\text { With 2 } \\
\text { aggregators } \\
\text { at }(15,50) \\
\text { and }(85,50)\end{array}$ & $\begin{array}{c}\text { With 2 } \\
\text { aggregators } \\
\text { at }(35,50) \\
\text { and }(65,50)\end{array}$ \\
\hline 3 & 97 & 97 & 98 \\
\hline 4 & 90 & 91 & 89 \\
\hline 5 & 71 & 72 & 73 \\
\hline 6 & 58 & 57 & 52 \\
\hline 7 & 43 & 43 & 42 \\
\hline 8 & 32 & 30 & 31 \\
\hline 9 & 21 & 20 & 22 \\
\hline
\end{tabular}

Table 4: Alive nodes comparison for different placement of aggregators

\begin{tabular}{|c|c|c|c|}
\hline $\begin{array}{c}\text { SEP } \\
\text { Heter } \\
\frac{\text { ogene }}{\text { ity }}\end{array}$ & $\begin{array}{c}\text { Alive nodes } \\
\text { with 2 } \\
\text { aggregators } \\
\text { at } \\
(25,50) \text { and } \\
(75,50)\end{array}$ & $\begin{array}{c}\text { With 2 } \\
\text { aggregators at } \\
(15,50) \text { and } \\
(85,50)\end{array}$ & $\begin{array}{c}\text { With 2 } \\
\text { aggregators at } \\
(35,50) \text { and } \\
(65,50)\end{array}$ \\
\hline 3 & 3 & 3 & 2 \\
\hline 4 & 10 & 9 & 11 \\
\hline 5 & 29 & 28 & 27 \\
\hline 6 & 42 & 43 & 48 \\
\hline 7 & 57 & 57 & 58 \\
\hline 8 & 68 & 70 & 69 \\
\hline 9 & 79 & 80 & 78 \\
\hline
\end{tabular}

Table 5: First Dead Node comparison for different placement of aggregators

\begin{tabular}{|c|c|c|c|}
\hline $\begin{array}{l}\text { SEP } \\
\text { Heter } \\
\frac{\text { ogenei }}{\underline{\text { ty }}}\end{array}$ & $\begin{array}{c}\text { First dead with } \\
\text { two } \\
\text { aggregators at } \\
(25,50) \text { and } \\
(75,50) \\
\end{array}$ & $\begin{array}{c}\text { First dead with } \\
\text { two } \\
\text { aggregators at } \\
(15,50) \text { and } \\
(85,50)\end{array}$ & $\begin{array}{c}\text { With } 2 \\
\text { aggregators at } \\
(35,50) \text { and } \\
(65,50)\end{array}$ \\
\hline 3 & 870 & 806 & 895 \\
\hline 4 & 1047 & 903 & 1117 \\
\hline 5 & 1311 & 1207 & 1067 \\
\hline 6 & 1476 & 1530 & 1599 \\
\hline 7 & 1869 & 1900 & 1866 \\
\hline 8 & 1909 & 1959 & 1920 \\
\hline 9 & 1967 & 2090 & 1942 \\
\hline
\end{tabular}

Table 6: Packets transmitted comparison for different placement of aggregators

\begin{tabular}{|c|c|c|c|}
\hline $\begin{array}{c}\text { SEP } \\
\text { Heterogeneity }\end{array}$ & $\begin{array}{c}\text { Packets to } \\
\text { Base } \\
\text { Station } \\
\text { with two } \\
\text { aggregators } \\
\text { at }(25,50) \\
\text { and }(75,50)\end{array}$ & $\begin{array}{c}\text { Packets to } \\
\text { Base } \\
\text { Station } \\
\text { with two } \\
\text { aggregators } \\
\text { at }(15,50) \\
\text { and }(85,50)\end{array}$ & $\begin{array}{c}\text { With 2 } \\
\text { aggregators } \\
\text { at }(35,50) \\
\text { and }(65,50)\end{array}$ \\
\hline 3 & 1 & 1 & 0 \\
\hline 4 & 2 & 5 & 1 \\
\hline 5 & 14 & 8 & 10 \\
\hline 6 & 13 & 17 & 16 \\
\hline 7 & 27 & 22 & 7 \\
\hline 8 & 36 & 28 & 37 \\
\hline 9 & 37 & 32 & 21 \\
\hline
\end{tabular}

\section{RESULTS \& ANALYSIS}

Simulation for proposed protocol is done in a field with dimensions $100 \mathrm{~m} \times 100 \mathrm{~m}$ and 100 nodes deployed in specific zones with respect to their energy. Two aggregators and one sink are placed in the network field. The first order radio model $s$ used in SEP. MATLAB is used to implement the simulations. In this model there are normal nodes and nodes with a higher level of energy. The number of nodes which have energies higher than the normal nodes is 10 in each case. For example In case of level 1 hierarchical M-SEP, 10 nodes are of advanced level. Rest all are normal nodes. Similarly in level 2 there are 10 advanced nodes and 10 super advanced nodes. Rests of the nodes are normal nodes and so on.

The efficiency is compared of M-SEP for different levels for different placement of aggregators in below mention figures.

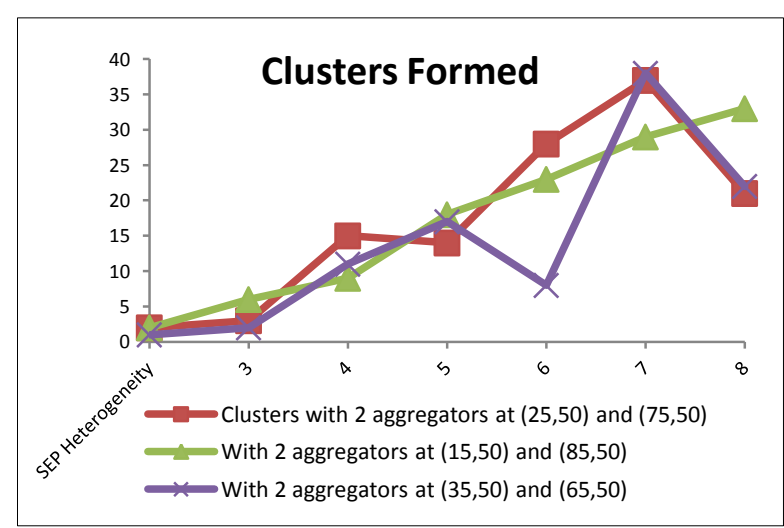

Fig 1: Clusters Formed with different placement of aggregators

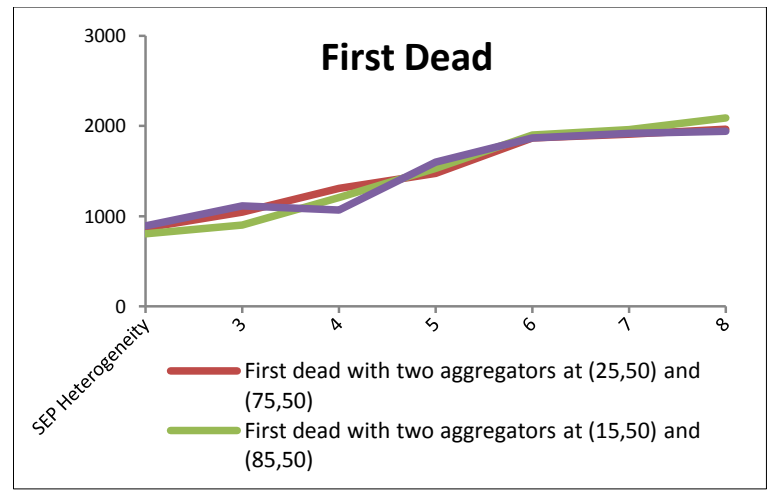

Fig 2: First dead node details

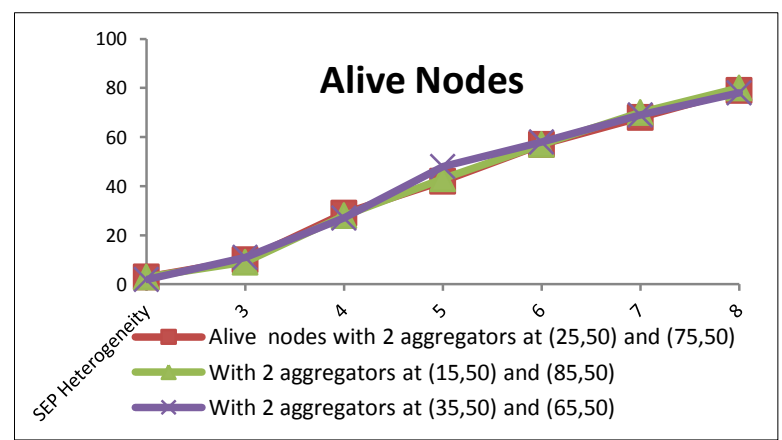

Fig 3: Alive nodes with different placement of aggregators 


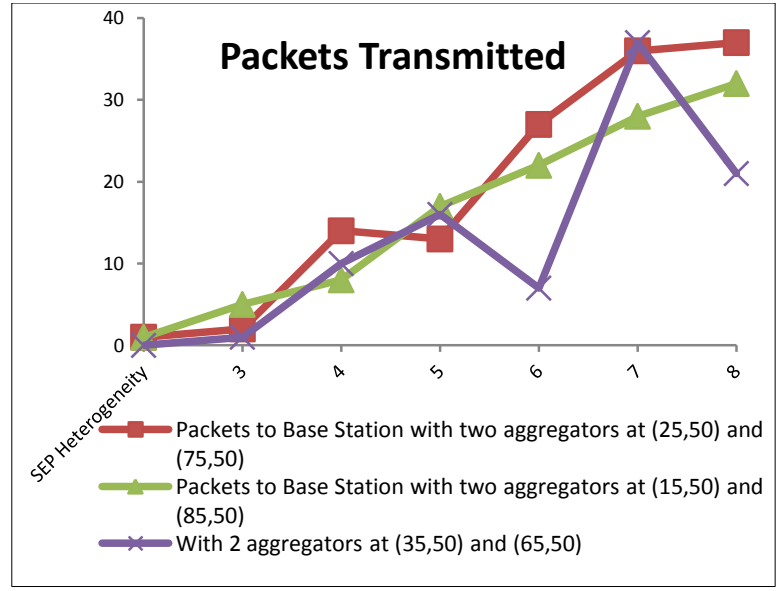

Fig 4: Packet transmission to base station

\section{CONCLUSION}

Here it is understood that as the level of heterogeneity increases:

- The number of clusters increases as can be seen with the upward trend

- The number of dead nodes at the end of 2500 rounds shows a decreasing trend

- The number of alive node increase with the level of heterogeneity

- The packets transmitted increase.

- First dead node also shows a delay with increasing heterogeneity.

This means that increasing the level of heterogeneity increases the network lifetime and efficiency of transmission and makes the wireless sensor network very efficient.

For aggregators ,the placement at $(25,50)$ and $(75,50)$ is the most favourable as from the simulation, as this placement shows the most steady trend in all parameters .This result can be derived analytically from theory too. Because this position is geometrically more equidistant from the cluster heads formed on the right and left side of the sink. Aggregators result in lower energy dissipation of the nodes, as cluster heads select which aggregator is near and compares its distance with that of sink. The one that is the nearest is used by the cluster heads to sink the information.
However, it can be seen that even the placement at $(25,50)$ and $(75,50)$ does not show a steady trend in the parameter- clusters formed. The trend of different parameters depends on the current random network distribution. Hence it can be said that the two aggregators on the sides of the sink leads to improved network lifetime and efficiency. But the different placement of these does not show any remarkable differences.

M-SEP with 9 levels of heterogeneity and two aggregators can be used in modern day heterogeneous wireless sensor networks for better transmission and network efficiency.

\section{REFERENCES}

[1] W.B. Heinzelman, A.P.Chandrakasan,and H. Balakrishnan,"An application specific protocol architecture for wireless microsensor networks"IEEE Transaction on wireless communication, vol 1, no. 4,pp.660-670,2002.

[2] G. Smaragdakis, I. Matta, A. Bestavros, "SEP: A Stable Election Protocol for clustered heterogeneous wireless sensor networks", Second International Workshop on Sensor and Actor Network Protocols and Applications (SANPA 2004), (2004)

[3] S. Faisal, N. Javaid, A. Javaid, M.A. Khan, S. H. Bouk, Z.A. Khan "Z-SEP: Zonal stable Election Protocol for wireless Sensor Networks",journal of Basic and applied scientific research ,(JBASR) March 2013.

[4] Said Benkirane, Abderrahim Benihssane, M. Lahcen. Hasanouli, Mohanmad Laghir "Distance based stable election protocol (DB-SEP) for heterogeneous wireless sensor network" International Journal of computer applications 58 (16): 9-15 November 2012.

[5] S. Bandhopadhyay, E.J. Coyle, "An energy efficient Hierarchical Clustering algorithm for wireless sensor networks," in : Proceeding of INFOCOM 2003, April 2003.

[6] O.Younis, S. fahmy, HEED: A hybrid, energy efficient distributed clustering approach for ad hoc sensor networks, IEEE transactions on Mobile computing 3(4) (2004) 660-669.

[7] F. Akyilidiz , W. Su, Y. Sankarasubramaniyam, E. Cayirici, "A survey on sensor networks", IEEE communications magazine 40 (80) (2002) 102-114. 